\title{
Absence of human papilloma virus in cervical adenocarcinoma determined by in situ hybridisation
}

\author{
F I Young, L M Ward, L J R Brown
}

\begin{abstract}
A few studies using DNA technology have suggested that human papillomavirus (HPV) may be an aetiological factor for adenocarcinoma of the uterine cervix. Twenty one cases of cervical adenocarcinoma were studied by in situ hybridisation using biotinylated DNA probes for HPV types 6, 11, 16 and 18 and a streptavidin, biotinylated alkaline phosphatase detection system. Intranuclear HPV DNA was detected in none of the adenocarcinomas, while positive controls gave a clear intranuclear signal. Adjacent areas of normal, koilocytic, and dysplastic squamous epithelium also gave positive results. It may be that squamous epithelium contaminates adenocarcinomas reported as positive by Southern blotting. Our results showing absence of detectable HPV DNA within adenocarcinomas suggest that HPV infection may not have a major role in the aetiology of adenocarcinoma of the uterine cervix.
\end{abstract}

The risk factors for invasive adenocarcinoma of the cervix uteri are less well understood than those of invasive squamous cell carcinoma where there is strong evidence that human papillomavirus (HPV) is the transmissible aetiological agent. ${ }^{1}$ Several studies have now linked adenocarcinoma of the cervix with HPV infection but most of these used Southern blotting allowing contamination from adjacent cervical intraepithelial neoplasia to take place. ${ }^{2}$ We used in situ hybridisation in an attempt to overcome this problem.

\section{Methods}

Single representative paraffin wax processed blocks from 21 cases of endocervical adenocarcinoma were retrieved from the file. Positive controls consisted of cases of benign cervical warts for HPV 6 and 11 and CIN positive for HPV 16. DNA probes to HPV type $6,11,16$ and 18 were biotinylated using the random primer method. Sections $5 \mu \mathrm{m}$ thick on slides pretreated with 3 aminopropyltriethoxysilane were pretreated with proteinase $\mathrm{K}$, heat denatured, and hybridised overnight with each HPV probe. A negative control with the probe omitted was included in each run. Hybridisation signal was detected using streptavadin, biotinylated alkaline phosphatase, and napthol AS-Bl phosphate.

\section{Results}

No intranuclear HPV DNA was identified in any of the 21 endocervical adenocarcinomas while positive controls showed intranuclear positivity in squamous cells. Seven test cases exhibited positivity for HPV in squamous epithelium: three areas of normal epithelium and two areas of koilocytosis contained HPV 11; one case showed both HPV 6 and 11 in koilocytes and another case showed HPV 18 in CIN with HPV 11 in adjacent normal epithelium.

\section{Discussion}

DNA hybridisation techniques, originally Southern blotting, identified HPV types 6,11 , 16,18 and 31 in CIN and squamous carcinoma of the uterine cervix. This supports epidemiological, morphological, and immunohistochemical findings suggesting that HPV has a role in the development of squamous neoplasia.

Adenocarcinoma accounts for $3-16 \%$ of cases of cervical carcinoma and its incidence seems to be increasing, ${ }^{1}$ either due to a reduction in development of invasive squamous carcinoma or due to a real increase secondary to exposure to an infective aetiological agent. It has been suggested that squamous carcinoma and adenocarcinoma have common aetiological factors and arise from a common stem cell, the subcolumnar reserve cell. ${ }^{3}$

HPV DNA was initially detected by Southern blotting in adenocarcinoma, ${ }^{12}$ but the samples may have been contaminated with $\mathrm{HPV}$ from adjacent $\mathrm{CIN}^{2}$ which is a common association. ${ }^{3}$ Two studies from the USA and one from Australia identified HPV by in situ hybridisation in $42.5 \%(17 / 40)$ of cervical adenocarcinomas $^{4}$ and in $70-88.6 \%$ (7/10 and $15 / 17)$ of in situ cervical adenocarcinomas. ${ }^{56}$ In contrast, a study from the United Kingdom identified HPV DNA by in situ hybridisation in only $6.3 \%(1 / 16)$ of invasive adenocarcinomas and $12.5 \%(1 / 8)$ of in situ adenocarcinomas (Griffin NR, Walker D, Lewis FA, Wells $M$, personal communication). These workers showed increased detection of HPV using the polymerase chain reaction (PCR) but this technique does not obviate the problem of contamination from adjacent squamous epithelium. The alarmingly high rates of HPV detection by PCR in normal cervices casts doubt on the clinical importance of these findings.

Our results seem to agree with the low incidence of HPV DNA detected in situ in the 
other United Kingdom study, suggesting that HPV may have on:y a limited role in causing adenocarcinoma of the uterine cervix.

1 Wilczynski SP, Walker J, Liao S, Bergen S, Berman M Adenocarcinoma of the cervix associated with human papillomavirus. Cancer 1988;62:1331-6.

2 Smotkin D, Berek JS, Fu YS, Hacker WF, Major FI, Wettstein FO. Human papillomavirus deoxynibonucleic Weid in the uterine cervix. Obstet Gynecol 1986;68:241-4.
3 Wells M, Brown LJR. Glandular lesions of the uterine cervix: the present state of our knowledge. Histopathol 1986;10:777-92.

4 Tase T, Okagaki T, Clark BA, et al. Human papillomavirus types and localisation in adenocarcinoma and adenosquamous carcinoma of the uterine cervix: A study by in situ DNA hybridisation. Cancer Res 1988;48:993-8.

5 Tase T, Okagaki T, Clark BA, Twiggs LB, Ostrow RS, Faras AJ. Human papillomavirus DNA in adenocarcinoma in situ microinvasive adenocarcinoma of the uterine cervix and coexisting cervical squamous intraepithelial neoplasia. Int J Gynecol Pathol 1989;8:8-17.

6 Farnsworth A, Laverly C, Stoler MH. Human papillomavirus messenger RNA expression in adenocarcinoma in situ of the uterine cervix. Int J Gynecol Pathol 1989;4: 321-30.

\title{
Persistent polyclonal B lymphocytosis with Epstein-Barr virus antibodies and subsequent malignant pulmonary blastoma
}

\author{
E Lawlor, M Murray, D S O'Briain, C Blaney, L Foroni, P Sarsfield, D Condell, \\ F Sullivan, S R McCann
}

Department of Haematology, St James's Hospital, Dublin 8, Ireland E Lawlor

M Murray

F Sullivan

C Blaney

P Sarsfield

S R McCann

Department of Histopathology, St James's Hospital D S O'Briain D Condell

Laboratory of

Molecular Genetics, Department of Haematology,

Hammersmith

Hospital, London

L Foroni

Accepted for publication 23 October 1990

\begin{abstract}
Persistent polyclonal B lymphocytosis (PPBL), a rare benign lymphoproliferative disorder, occurred in a 25 year old woman. The lymphocytes showed the phenotype cIgM +, FMC7 +, CD19+, CD20 +, which was similar to that seen in Waldenstrom's macroglobinaemia, but the proliferation was repeatedly shown to be polyclonal both immunologically and by immunoglobulin gene rearrangement studies. Eleven years after presentation the patient developed a malignant pulmonary blastoma, a rare pulmonary tumour of combined epithelial and mesenchymal origin.

The failure to develop a lymphoid malignancy over 11 years together with immunological and genetic evidence of polyclonality confirms that PPBL is a benign lymphoproliferative disorder. The subsequent occurrence of a nonhaemopoietic malignancy suggests that benign lymphoid proliferations, like their malignant counterparts, may predispose to the development of neoplasia. A role for Epstein-Barr virus in the pathogenesis of one or both of these rare conditions is suggested by the finding of raised Epstein-Barr viral capsid antigen titres.
\end{abstract}

Persistent polyclonal B lymphocytosis (PPBL) is a rare disorder first described in 1982 in three patients in whom a polyclonal $B$ cell lymphocytosis with characteristic binucleate and atypical forms persisted in peripheral blood for months to years. ${ }^{1}$ The three patients were all female, middle aged, smokers, and homozygous for HLA DR 7; they had recurrent chest infections and dysgammaglobulinaemia. We report PPBL in a patient with positive Epstein-Barr virus (EBV) serology who subsequently developed a malignant pulmonary blastoma.

\section{Case report}

A 43 year old woman had had recurrent chest infections since 1976 and persistent lymphocytosis $\left(10-17.5 \times 10^{9} / 1\right)$ with binucleate and other atypical forms (fig 1A). Intermittently, splenomegaly and lymphadenopathy were present. Immunological studies in 1982 and 1986 showed a polyclonal B cell proliferation (CD19 (75\%), CD20 (40\%), FMC7 $(67 \%), \operatorname{cIgM}(65 \%), \kappa(50 \%), \lambda(30 \%))$ similar to that described in Waldenstrom's macroglobinaemia, ${ }^{2}$ but here repeatedly shown to be polyclonal. Immunoglobulin gene rearrangement studies did not detect any rearranged bands. There was a polyclonal rise in serum $\operatorname{IgM}(5.8 \mathrm{~g} / \mathrm{l})$ with low normal IgA and IgG concentrations. Repeated monospot tests were negative but an Epstein-Barr IgG viral capsid antigen (VCA) (October 1986) was positive (1/1280); Epstein-Barr IgM VCA was negative. Other viral studies for HIV, HTLVI, and human B lymphotropic virus were negative. The patient was a cigarette smoker and her HLA DR type was DR1 DR7.

In October 1986 she presented with a dry 